\title{
The Gradient Theory of Phase Transitions and the Minimal Interface Criterion
}

\author{
LUCIANO MODICA
}

\author{
Communicated by M. E. GURTIN
}

\section{Introduction}

In this paper I prove some conjectures of GURTIN [15] concerning the Van der Waals-Cahn-Hilliard theory of phase transitions.

Consider a fluid, under isothermal conditions and confined to a bounded container $\Omega \subset \mathbb{R}^{n}$, whose Gibbs free energy, per unit volume, is a prescribed function $W_{0}$ of the density distribution $u$. The classical problem (cf. GuRTIN [16]) of determining the stable configurations of the fluid is to minimize the total energy of the fluid,

$$
E(u)=\int_{\Omega} W_{0}(u(x)) d x,
$$

among all density distributions $u$ of prescribed total mass $m$ :

$$
\int_{\Omega} u(x) d x=m .
$$

Suppose that $W_{0}$ acts as in Figure 1, with two relative minima.

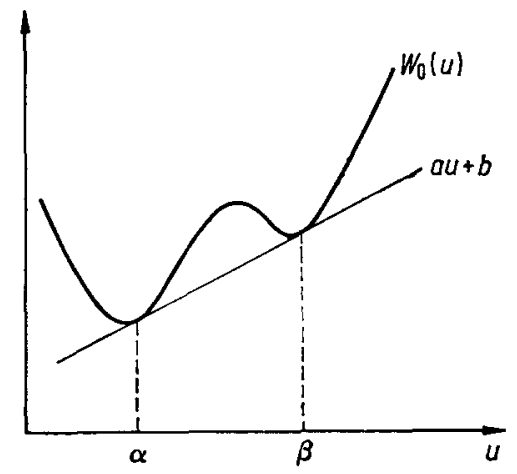

Fig. 1 
It is obvious that the foregoing minimization problem remains unchanged when $W_{0}(u)$ is replaced by $W(u)=W_{0}(u)-(a u+b)$. (Of course, the minimum value changes by a constant). If $\alpha$ and $\beta$ denote the (absolute) minimizers of $W$, such a problem admits only piecewise constant solutions with values $\alpha$ or $\beta$. Moreover, for $\alpha|\Omega|<m<\beta|\Omega|$, there are infinitely many such solutions, with no restriction on the shape of the interface between the sets $\{u=\alpha\}$ and $\{u=\beta\}$. In particular, there is no way to recover the physically reasonable criterion that the interface have minimal area.

To overcome difficulties of this type, the Van der Waals-Cahn-Hilliard ${ }^{1}$ theory is based on the energy functional

or, equivalently,

$$
E_{\varepsilon}(u)=\int_{\Omega}\left[\varepsilon|D u|^{2}+W_{0}(u)\right] d x
$$

$$
\mathscr{E}_{\varepsilon}(u)=\int_{\delta}\left[\varepsilon|D u|^{2}+W(u)\right] d x
$$

in which interfacial energy is modeled by the dependence on the density gradient, and where $\varepsilon>0$ is a small parameter. The mathematical problem is then to study the asymptotic behavior, as $\varepsilon \rightarrow 0^{+}$, of solutions $u_{\varepsilon}$ of the minimization problem

and to prove that

$$
\min \left\{\mathscr{E}_{\varepsilon}(u): \int_{\Omega} u(x) d x=m\right\}
$$

$\left(^{*}\right) \quad\left(u_{\varepsilon}\right)$ converges to a function $u_{0}$ which takes only the values $\alpha$ and $\beta$ with interface between the sets $\left\{u_{0}=\alpha\right\}$ and $\left\{u_{0}=\beta\right\}$ having minimal area.

In this paper we establish (*) (modulo replacing $\left(u_{\varepsilon}\right)$ by a subsequence) for arbitrary dimension ${ }^{2} n \geqq 1$, for any bounded, open set $\Omega$ with Lipschitz continuous boundary, and under very mild assumptions on $W$ ( $c f$. Theorem I and Proposition 3 in section 2). A similar result has been announced by R. V. KoHN and P. STERnBERG.

The plan of the paper is as follows: in section 1, I give some lemmas on sets with bounded perimeter in the sense of CACCIOPPOLI and DE GIORGI; in section 2, I state and prove the main results; in section 3 , I mention the possibility of including my results within the theory of $\Gamma$-convergence.

It is worth noting that my proof very closely follows ideas of an old example of $\Gamma$-convergence ( $c f$. Modica \& MorToLa [20]) for functionals of essentially the same form as $\mathscr{E}_{\varepsilon}$. This example was proposed by E. DE GIORGI, within a completely different physical context. A generalization of this example ( $c f$. ModicA \& MoRTola [21]) was never published in the international literature because it seemed specialized and of little importance. It has been surprising for me to find

1 Cf. Carr, Gurtin \& Slemrod [5], Novick-Cohen \& Segel [22], Alikakos \& Shaing [1], Gurtin \& Matano [17], Gurtin [16], AlmGren \& Gurtin [2], which may be consulted for a more complete bibliography and for details of the thermodynamic setting.

$2 C f$. Carr, Gurtin \& Slemrod [5], who show, for the special case $n=1$, that the limiting interface is a single point. 
these techniques important in solving a completely different problem from thermodynamics. For this, I am indebted to R. V. KoHN and P. PoDio Guidugli, who informed me of GuRTIN's results. I also wish to thank M. E. GURTIN for his interest.

\section{Sets with bounded perimeter}

In this section we recall some properties of sets with bounded perimeter and prove some lemmas used later in the proof of the main results. The standard references for this subject are the original papers by DE GIORGI [6], [7] and the books by Federer [10], Giusti [13], Massari \& Miranda [19].

For any open subset ${ }^{3} \Omega$ of $\mathbb{R}^{n}$ and for every $u \in L_{\text {loc }}^{1}(\Omega)$, we define

$$
\int_{\Omega}|D u|=\sup \left\{\int_{\Omega} u(x) \sum_{i=1}^{n}\left(D_{i} g_{i}\right)(x) d x: g \in C_{0}^{\infty}\left(\Omega, \mathbb{R}^{n}\right),|g| \leqq 1\right\} .
$$

If $\int_{\Omega}|u| d x<+\infty$ and $\int_{\Omega}|D u|<+\infty$, we write $u \in B V(\Omega)$. Note that the Sobolev space $\mathscr{W}^{1,1}(\Omega)$ is contained in $B V(\Omega)$ and $\int_{\Omega}|D u|$ equals, for $u \in \mathscr{W}^{1,1}(\Omega)$, the ordinary Lebesgue integral $\int_{\Omega}|D u(x)| d x$. There are, however, functions in $B V(\Omega) \backslash \mathscr{W}^{1,1}(\Omega) ;$ e.g., the characteristic functions of the smooth, open, bounded subsets of $\Omega$.

It is obvious that, if $\left(u_{h}\right)$ is a sequence in $L^{1}(\Omega)$ which converges in $L^{1}(\Omega)$ to $u_{\infty}$, then

$$
\int_{\Omega}|D u| \leqq \liminf _{h \rightarrow+\infty} \int_{\Omega}\left|D u_{h}\right|
$$

Moreover, one can prove that the sets

$$
\left\{u \in L^{1}(\Omega): \int_{\Omega}|u| d x+\int_{\Omega}|D u| \leqq c\right\}
$$

are compact in $L^{1}(\Omega)$, for every real constant $c$, provided that $\Omega$ is bounded. Each $u \in B V(\Omega)$ can be approximated by a sequence $\left(u_{h}\right)$ in $C^{\infty}(\Omega)$ in the sense that (cf. Anzellotti \& Giaquinta [3])

$$
\lim _{h \rightarrow+\infty}\left[\int_{\Omega}\left|u_{h}-u\right| d x+\left|\int_{\Omega} D u_{h}\right|-\int_{\Omega}|D u| \mid\right]=0
$$

hence, by the Poincare inequality, if $\Omega$ is bounded, then

(2) the sets $\left\{u \in L^{1}(\Omega): \int_{\Omega} u=m, \int_{\Omega}|D u| \leqq c\right\}$ are compact in $L^{1}(\Omega)$ whenever $m$ and $c$ are real constants. tions.

All results are stated for $n \geqq 2$; they hold also for $n=1$ with obvious modifica- 
Let us fix an open subset $\Omega$ of $\mathbb{R}^{n}$ and $u \in B V(\Omega)$. Then the map $A \rightarrow \int_{A}|D u|$, defined for any open subset $A$ of $\Omega$, is the trace on the open sets of a uniquely determined Borel measure on $\Omega$; we shall denote by $\int_{E}|D u|$ the value of this
measure on a measurable subset $E$ of $\Omega$.

Henceforth, $\mathscr{H}_{n-1}$ will denote the $(n-1)$-dimensional Hausdorff measure and $|\cdot|$ the Lebesgue measure on $\mathbb{R}^{n}$. Let $\Omega$ be an open subset of $\mathbb{R}^{n}$ with Lipschitz continuous boundary. Then

$$
\begin{gathered}
\forall u \in B V(\Omega) \cap L^{\infty}(\Omega) \quad \exists \tilde{u} \in B V\left(\mathbb{R}^{n}\right) \cap L^{\infty}\left(\mathbb{R}^{\mathrm{n}}\right): \\
u=\tilde{u} \quad \text { on } \Omega, \quad \int_{\partial \Omega}|D \tilde{u}|=0 .
\end{gathered}
$$

The assumption that $\partial \Omega$ is Lipschitz continuous could be weakened ( $c f$. ANZELlotTI \& GIAQUiNTA [3]), but it does not seem sufficient to assume only that $\mathscr{H}_{n-1}(\partial \Omega)<+\infty$.

If $E$ is any measurable subset of $\mathbb{R}^{n}$, we denote by $I_{E}$ the characteristic function of $E$ and, for every open subset $\Omega$ of $\mathbb{R}^{n}$, we let

$$
P_{\Omega}(E)=\int_{\Omega}\left|D I_{E}\right|
$$

If $P_{\Omega}(E)<+\infty$, we say that $E$ has bounded perimeter in $\Omega$. It can be proved that $P_{\Omega}(E) \leqq \mathscr{H}_{n-1}(\partial E \cap \Omega)$ with equality if, for instance, $\partial E \cap \Omega$ is a Lipschitz continuous hypersurface.

Let $\Omega$ be an open subset of $\mathbb{R}^{n}$ and $u \in B V\left(\mathbb{R}^{n}\right)$. Then the function $t \rightarrow$ $P_{\Omega}\left(\left\{x \in \mathbb{R}^{n}: u(x)>t\right\}\right)$ is Lebesgue measurable on $\mathbb{R}$ and the Fleming-Rishel [11] formula holds:

$$
\int_{\Omega}|D u|=\int_{-\infty}^{+\infty} P_{\Omega}\left(\left\{x \in \mathbb{R}^{n}: u(x)>t\right\}\right) d t .
$$

We now state and prove four lemmas. The first asserts that every set with bounded perimeter in $\Omega$ can be approximated in volume and in perimeter by a sequence of smooth subsets of $\mathbb{R}^{n}$, all having the same volume inside $\Omega$ and each of whose boundaries satisty a measure-theoretic transversality condition with respect to $\partial \Omega$.

Lemma 1. Let $\Omega$ be an open, bounded subset of $\mathbb{R}^{n}$ with Lipschitz continuous boundary, and let $E$ be a measurable subset of $\Omega$. If $E$ and $\Omega \backslash E$ both contain a nonempty open ball, then there exists a sequence $\left(E_{h}\right)$ of open bounded subsets of $\mathbb{R}^{n}$ with smooth boundaries such that

(i) $\lim _{h \rightarrow+\infty}\left|\left(E_{h} \cap \Omega\right) \triangle E\right|=0, \quad \lim _{h \rightarrow+\infty} P_{S}\left(E_{h}\right)=P_{\Omega}(E)$;

(ii) $\left|E_{h} \cap \Omega\right|=|E|$ for $h$ large enough;

(iii) $\mathscr{H}_{n-1}\left(\partial E_{h} \cap \partial \Omega\right)=0$ for $h$ large enough .

Proof. Let $u=I_{E}$ and, by (3), let $\tilde{u} \in B V\left(\mathbb{R}^{n}\right) \cap L^{\infty}\left(\mathbb{R}^{n}\right)$ be such that $\tilde{u}=u$ on $\Omega$ and $\int_{\partial \Omega}|D \tilde{u}|=0$. Let $\left(\phi_{\varepsilon}\right)$ be the usual system of mollifiers: $\phi_{\varepsilon} \in C_{0}^{\infty}\left(\mathbb{R}^{n}\right)$; 

spt $\phi_{\varepsilon} \leqq B(0, \varepsilon) ; 0 \leqq \phi_{\varepsilon} \leqq 1 ; \int_{\mathrm{R}^{n}} \phi_{\varepsilon} d x=1$. Then, defining $u_{\varepsilon}=u * \phi_{\varepsilon}$, we
easily infer that

$$
\lim _{\varepsilon \rightarrow 0^{+}} \int_{\mathrm{R}^{n}}\left|u_{\varepsilon}-\tilde{u}\right| d x=0
$$

hence

$$
\lim _{\varepsilon \rightarrow 0^{+}}\left|\left\{x \in \mathbb{R}^{n}:\left|u_{\varepsilon}(x)-\tilde{u}(x)\right| \geqq \eta\right\}\right|=0 \quad \forall \eta>0
$$

and, moreover,

$$
\lim _{\varepsilon \rightarrow 0^{+}} \int_{\mathrm{R}^{n}}\left|D u_{\varepsilon}\right| d x=\int_{\mathrm{R}^{n}}|D \tilde{u}|
$$

From this last equality and the identity $\int_{\partial \Omega}|D \tilde{u}|=0$ we conclude, by applying the lower semicontinuity (1) to $\Omega$ and to $\mathbb{R}^{n} \backslash \bar{\Omega}$, that

$$
\lim _{\varepsilon \rightarrow 0^{+}} \int_{\Omega}\left|D u_{\varepsilon}\right| d x=\int_{\Omega}|D \tilde{u}|=\int_{\Omega}|D u|=P_{\Omega}(E) .
$$

The idea of the proof is now clear: after approximating $u=I_{E}$ by smooth functions $\left(u_{\varepsilon}\right)$, we want to pass to a sequence $\left(E_{\varepsilon}\right)$ of sets, which approximate $E$, by choosing suitable level sets of $u_{\varepsilon}$. Unfortunately, the proof is technical.

Recall that, by the hypotheses, there exist $x_{1} \in E, x_{2} \in \Omega \backslash E, \delta_{0}>0$ such that

so that

$$
B_{1}=B\left(x_{1}, \delta_{0}\right) \subset E, \quad B_{2}=B\left(x_{2}, \delta_{0}\right) \subset \Omega \backslash E,
$$

$$
u_{\varepsilon}=u \quad \text { on } B_{1} \cup B_{2} \quad \text { for every } \varepsilon<\frac{\delta_{0}}{2}
$$

Now, for every $h \in \mathbb{N}$, let us choose a positive number $\varepsilon_{h}<\min \left\{1 / h, \delta_{0} / 2\right\}$ such that (see (5))

$$
\left|\left\{x \in \Omega:\left|u_{\varepsilon_{h}}(x)-u(x)\right| \geqq \frac{1}{h}\right\}\right| \leqq \frac{1}{h} .
$$

Moreover, writing

$$
v_{h}=\operatorname{essinf}_{1 / h \leqq t \leqq 1-1 / h} P_{s}\left(\left\{x \in \mathbb{R}^{n}: u_{\varepsilon_{h}}(x)>t\right\}\right),
$$

(essinf is the essential infimum of a Lebesgue measurable function), we choose $\left.t_{h} \in\right] \frac{1}{h}, 1-\frac{1}{h}[$ such that

$$
\begin{gathered}
P_{\Omega}\left(\left\{x \in \mathbb{R}^{n}: u_{\varepsilon_{h}}(x)>t_{h}\right\}\right) \leqq v_{h}+\frac{1}{h}, \\
D u_{\varepsilon_{h}}(x) \neq 0 \quad \forall x \in \mathbb{R}^{n}: u_{\varepsilon_{h}}(x)=t_{h}, \\
\mathscr{H}_{n-1}\left(\left\{x \in \partial \Omega: u_{\varepsilon_{h}}(x)=t_{h}\right\}\right)=0 .
\end{gathered}
$$


Note that (9) holds for a set of $t_{h}$ with positive measure, so (10) and (11) can be fulfilled by appealing, respectively, to Sard's Lemma and to $\mathscr{H}_{n-1}(\partial \Omega)<+\infty$.

Finally, using $\varepsilon_{h}$ and $t_{h}$, we can construct $E_{h}$ by setting

$$
\begin{gathered}
\tilde{E}_{h}=\left\{x \in \mathbb{R}^{n}: u_{\varepsilon_{h}}(t)>t_{h}\right\}, \\
\lambda_{h}=\left|\tilde{E}_{h} \cap \Omega\right|-|E|, \\
E_{h}= \begin{cases}\tilde{E}_{h} \backslash B\left(x_{1}, r_{h}\right) & \text { if } \lambda_{h}>0 \\
\tilde{E}_{h} & \text { if } \lambda_{h}=0 \\
\tilde{E}_{h} \cup B\left(x_{2}, r_{h}\right) & \text { if } \lambda_{h}<0,\end{cases}
\end{gathered}
$$

where $r_{h}$ is chosen such that $\left|B\left(x_{1}, r_{h}\right)\right|=\left|B\left(x_{2}, r_{h}\right)\right|=\left|\lambda_{h}\right|$.

We first prove that $\left|E_{h} \cap \Omega\right|=|E|$. Note that

while

$$
x \in\left(\tilde{E}_{h} \cap \Omega\right) \backslash E \Rightarrow u_{e_{h}}(x)>t_{h}>\frac{1}{h} \quad \text { and } \quad u(x)=0
$$

$$
x \in E \backslash\left(\tilde{E}_{h} \cap \Omega\right) \Rightarrow u_{\varepsilon_{h}}(x) \leqq t_{h}<1-\frac{1}{h} \quad \text { and } \quad u(x)=1,
$$

so we conclude, by ( 8$)$, that

$$
\left|\lambda_{h}\right| \leqq\left(\tilde{E}_{h} \cap \Omega\right) \Delta E|\leqq|\left\{x \in \Omega:\left|u_{\varepsilon_{h}}(x)-u(x)\right| \geqq \frac{1}{h}\right\} \mid \leqq \frac{1}{h} ;
$$

hence, by the definition of $r_{h}$,

$$
\lim _{h \rightarrow+\infty} r_{h}=0
$$

It follows that, for $h$ large enough, $r_{h}<\delta_{0}$; hence $\overline{B\left(x_{1}, r_{h}\right)} \subset B_{1}, \overline{B\left(x_{2}, r_{h}\right)} \subset B_{2}$. On the other hand, as $e_{h}<\delta_{0} / 2$, we obtain, by (7), that

$$
B_{1} \subseteq \tilde{E}_{h} \cap \Omega, \quad B_{2} \leqq \Omega \backslash \tilde{E}_{h},
$$

and therefore

$$
\begin{aligned}
& \left|E_{h} \cap \Omega\right|=\left|\tilde{E}_{h} \cap \Omega\right|-\left|B\left(x_{1}, r_{h}\right)\right|=|E| \quad \text { if } \lambda_{h}>0 \\
& \left|E_{h} \cap \Omega\right|=\left|\tilde{E}_{h} \cap \Omega\right|+\left|B\left(x_{2}, r_{h}\right)\right|=|E| \quad \text { if } \lambda_{h}<0
\end{aligned}
$$

hence (ii) is proved.

Analogously,

$$
\partial E_{h} \cap \partial \Omega=\left(\partial \tilde{E}_{h} \cup \partial B\left(x_{i}, r_{h}\right)\right) \cap \partial \Omega \quad \text { for } i=1 \text { or } i=2 \text {; }
$$

as $\partial B\left(x_{i}, r_{h}\right) \cap \partial \Omega=\emptyset$ for $i=1,2$, we conclude, by (11), that

$$
\mathscr{H}_{n-1}\left(\partial E_{h} \cap \partial \Omega\right)=\mathscr{H}_{n-1}\left(\partial \tilde{E}_{h} \cap \partial \Omega\right)=0
$$

which proves (iii). 
The sets $E_{h}$ are obviously bounded and, because of (10), have smooth boundaries. Thus, we have only to prove (i).

The fact that

$$
\lim _{h \rightarrow+\infty}\left|\left(E_{h} \cap \Omega\right) \triangle E\right|=0
$$

is a direct consequence of

$$
\left|\left(E_{h} \cap \Omega\right) \Delta\left(\tilde{E_{h}} \cap \Omega\right)\right|=\left|\lambda_{h}\right|
$$

in conjunction with (12).

Finally, to prove that

$$
\lim _{h \rightarrow+\infty} P_{\Omega}\left(E_{h}\right)=P_{\Omega}(E),
$$

we first observe that the argument used before yields

$$
P_{\Omega}\left(E_{h}\right)=P_{\Omega}\left(\tilde{E}_{h}\right)+\mathscr{H}_{n-1}\left(\partial B\left(x_{i}, r_{h}\right)\right)
$$

for $i=1$ or $i=2$ and for $h$ large enough, while (12) and the lower semicontinuity (1) give

$$
P_{\Omega}(E) \leqq \liminf _{h \rightarrow+\infty} P_{\Omega}\left(\tilde{E_{h}}\right) .
$$

For the converse inequality, we deduce from (9) that

$$
P_{\Omega}\left(\tilde{E}_{h}\right) \leqq \frac{1}{h}+v_{h} \leqq \frac{1}{h}+P_{\Omega}\left(\left\{x \in \mathbb{R}^{n}: u_{\varepsilon_{h}}(x)>t\right\}\right)
$$

for every $h \in \mathbb{N}$ and for almost all $t \in] \frac{1}{h}, 1-\frac{1}{h}[$, so, integrating in $t$ between $\frac{1}{h}$ and $1-\frac{1}{h}$ and applying the Fleming-Rishel formula (4), we obtain

$$
\left(1-\frac{2}{h}\right) P_{\Omega}\left(\tilde{E}_{h}\right) \leqq \frac{1}{h}\left(1-\frac{2}{h}\right)+\int_{\Omega}\left|D u_{\varepsilon_{h}}\right| d x \quad \forall h \in \mathbb{N} ;
$$

recalling that $\varepsilon_{h} \leqq \frac{1}{h}$, we may use (6) to conclude that

$$
\limsup _{h \rightarrow+\infty} P_{\Omega}\left(\tilde{E}_{h}\right) \leqq P_{\Omega}(E) .
$$

Finally, (13), (15), (16), and (17) yield the equality (14), and the proof of Lemma 1 is complete.

Our second lemma asserts that, to verify a minimum property for the perimeter, it suffices to restrict the class of competing sets to sets with smooth boundary.

Lemma 2. Let $\Omega$ be an open, bounded subset of $\mathbb{R}^{n}$ with Lipschitz continuous boundary, and let $E$ be a measurable subset of $\Omega$ with $0<|E|<|\Omega|$. If for a fixed $\lambda \geqq 0$ we have $\lambda \leqq \mathrm{P}_{\Omega}(\mathrm{A})$ for every open, bounded subset $A$ of $\mathbb{R}^{n}$ which has 
smooth boundary and satisfies $\mathscr{H}_{n-1}(\partial A \cap \partial \Omega)=0,|A \cap \Omega|=|E|$, then

$$
\lambda \leqq \min \left\{P_{\Omega}(F): F \text { measurable subset of } \Omega,|F|=|E|\right\} .
$$

If, in particular, $\lambda=P_{\Omega}(E)$, then equality holds.

Proof. Note that, because of the definition of $P_{\Omega}(F)$, the lower semicontinuity (1) and the compactness (2), there is a measurable subset $E_{0}$ of $\Omega$ such that

$$
P_{\Omega}\left(E_{0}\right)=\min \left\{P_{\Omega}(F): F \text { measurable subset of } \Omega,|F|=|E|\right\} .
$$

By a theorem of Gonzalez, Massari \& Tamanini ([14], th. 1), both $E_{0}$ and $\Omega \backslash E_{0}$ contain a non-empty open ball, so by the hypotheses and Lemma 1 we conclude that $\lambda \leqq P\left(E_{0}\right)$, and Lemma 2 is proved.

The third lemma of this section discusses a geometrically evident property of the tubular neighborhoods of a compact smooth hypersurface in $\mathbb{R}^{n}$.

Lemma 3. Let $A$ be an open subset of $\mathbb{R}^{n}$ with smooth, non-empty, compact boundary and define $g(x)=$ dist $(x, \partial A)$, and, for $t>0, S_{t}=\{x \in A: g(x)=t\}$. If $\Omega$ is an open subset of $\mathbb{R}^{n}$ such that $\mathscr{H}_{n-1}(\partial A \cap \partial \Omega)=0$, then

(i) $\lim _{t \rightarrow 0^{+}} \mathscr{H}_{n-1}\left(S_{t} \cap \Omega\right)=\mathscr{H}_{n-1}(\partial A \cap \Omega)$.

Proof. We first prove (i) with $\Omega=\mathbb{R}^{n}$. For $t>0$, let $V_{t}=\{x \in A: 0<g(x)<t\}$; by repeating an argument of GiLbarg \& TRUDINGer ([12], App. A), we find that, for $t$ small enough, there is a diffeomorphism $\phi$ between $V_{t}$ and $\partial A \times] 0, t[$ such that

$$
\operatorname{det}(D \phi)(x)=\prod_{i=1}^{n-1}\left(1-k_{i}(\hat{\phi}(x)) g(x)\right) \quad \forall x \in V_{t},
$$

where $k_{1}, \ldots, k_{n-1}$ denote the principal curvatures of $\partial A$ and $\hat{\phi}(x)$ denotes the component of $\phi(x)$ on $\partial A$. Moreover, $g$ is smooth on $\bar{V}_{t}$ and

$$
D g(x)=-v(\hat{\phi}(x)) \quad \forall x \in \bar{V}_{t},
$$

where $v$ denotes the outer normal vector to $\partial A$. Finally, if $v_{t}$ denotes the normal vector to $S_{t}$, outward with respect to $V_{t}$, we have

$$
v_{t}(x)=D g(x) \quad \forall x \in S_{t} .
$$

Now, the divergence theorem, (19) and (20) imply that

$$
\begin{aligned}
\int_{V_{t}} \Delta g(x) d x & =\int_{\partial A} D g \cdot v d \mathscr{H}_{n-1}+\int_{S_{t}} D g \cdot v_{t} d \mathscr{H}_{n-1} \\
& =\mathscr{H}_{n-1}\left(S_{t}\right)-\mathscr{H}_{n-1}(\partial A),
\end{aligned}
$$

and it is sufficient to verify that $\left|V_{t}\right|$ tends to zero as $t \rightarrow 0^{+}$. Indeed, by (18) and the smoothness and compactness of $\partial A$, det $D \phi(x) \geqq \mu>0$ for $x \in V$, 
and $t$ small enough; hence

$$
\begin{aligned}
\lim _{h \rightarrow 0^{+}}\left|V_{t}\right| & =\lim _{t \rightarrow 0^{+}} \int_{\partial A} d \mathscr{H}_{n-1}(y) \int_{0}^{t}\left[\operatorname{det} D \phi^{-1}(y, s)\right] d s \\
& \leqq \lim _{t \rightarrow 0^{+}} t \mu^{-1} \mathscr{H}_{n-1}(\partial A)=0,
\end{aligned}
$$

and (i) is proved when $\Omega=\mathbb{R}^{n}$.

We now remove this last condition. Note that $S_{t}=\partial\left(A \backslash V_{t}\right)$; hence, for $t$ small enough,

By (21),

$$
\mathscr{H}_{n-1}\left(S_{t} \cap \Omega\right)=P_{\Omega}\left(A \backslash V_{t}\right)
$$

$$
\lim _{t \rightarrow 0^{+}} \int_{\mathrm{R}^{n}}\left|I_{A}-I_{A \backslash V_{t}}\right| d x=\lim _{t \rightarrow 0^{+}} \int_{\mathrm{R}^{n}} I_{V_{t}} d x=0
$$

and the lower semicontinuity (1) yields

$$
\mathscr{H}_{n-1}(\partial A \cap \Omega)=P_{\Omega}(A) \leqq \liminf _{t \rightarrow 0^{+}} P_{\Omega}\left(A \backslash V_{t}\right)=\liminf _{t \rightarrow 0^{+}} \mathscr{H}_{n-1}\left(S_{t} \cap \Omega\right)
$$

On the other hand,

$$
\mathscr{H}_{n-1}\left(S_{t} \cap \Omega\right) \leqq \mathscr{H}_{n-1}\left(S_{t}\right)-\mathscr{H}_{n-1}\left(S_{t} \cap\left(\mathbb{R}^{n} \backslash \bar{\Omega}\right)\right)
$$

and, as before,

$$
\mathscr{H}_{n-1}\left(\partial A \cap\left(\mathbb{R}^{n} \backslash \bar{\Omega}\right)\right) \leqq \liminf _{t \rightarrow 0^{+}} \mathscr{H}_{n-1}\left(S_{t} \cap\left(\mathbb{R}^{n} \backslash \bar{\Omega}\right)\right)
$$

hence, for the hypothesis $\mathscr{H}_{n-1}(\partial A \cap \partial \Omega)=0$,

$$
\begin{aligned}
& \limsup _{t \rightarrow 0^{+}} \mathscr{H}_{n-1}\left(S_{t} \cap \Omega\right) \leqq \mathscr{H}_{n-1}(\partial A)-\mathscr{H}_{n-1}\left(\partial A \cap\left(\mathbb{R}^{n} \backslash \bar{\Omega}\right)\right) \\
& =\mathscr{H}_{n-1}(\partial A \cap \Omega) \text {. }
\end{aligned}
$$

The results (22) and (23) complete the proof of Lemma 3.

The last lemma is a two-sided version and an immediate corollary of Lemma 3.

Lemma 4. Let $A$ and $\Omega$ be as in Lemma 3 and define the function $h: \mathbb{R}^{n} \rightarrow \mathbb{R}$ by

$$
h(x)=\left\{\begin{aligned}
-\operatorname{dist}(x, \partial A) & \text { if } x \in A \\
\operatorname{dist}(x, \partial A) & \text { if } x \notin A .
\end{aligned}\right.
$$

Then $h$ is Lipschitz continuous, $|D h(x)|=1$ for almost all $x \in \mathbb{R}^{n}$ and, if $S_{t}=\left\{x \in \mathbb{R}^{n}: h(x)=t\right\}$,

(i) $\lim _{t \rightarrow 0} \mathscr{H}_{n-1}\left(S_{t} \cap \Omega\right)=\mathscr{H}_{n-1}(\partial A \cap \Omega)$.

Proof. It is obvious that $|h(x)-h(y)| \leqq|x-y|$ for every $x, y \in \mathbb{R}^{n}$; hence $h$ is Lipschitz continuous and $|D h(x)| \leqq 1$ for almost all $x \in \mathbb{R}^{n}$. Moreover, for 
every $x \in \mathbb{R}^{n} \backslash \partial A$ there exists $x_{0} \in \partial A$ such that $h(x)= \pm\left|x-x_{0}\right|$ and $x-x_{0}$ is orthogonal to $\partial A$ in $x_{0}$. Then, at each point $y$ of the segment $\left[x, x_{0}\right]$, we have $h(y)= \pm\left|y-x_{0}\right|$; hence $|h(x)-h(y)|=|x-y|$ for every $y \in\left[x, x_{0}\right]$, and $|D h(x)|=1$ for almost all $x \in \mathbb{R}^{n}$.

To prove (i) it suffices to apply Lemma 3 to $A$ and to $\mathbb{R}^{n} \backslash \bar{A}$.

\section{Main results}

Throughout this section $\Omega$ will be a fixed open, bounded subset of $\mathbf{R}^{n}$ with Lipschitz continuous boundary, and $W:[0,+\infty[\rightarrow \mathbb{R}$ will be a fixed non-negative, continuous function with exactly two zeros $\alpha, \beta \quad(0<\alpha<\beta)$. For every $\varepsilon>0$ and every $u \in L^{1}(\Omega)$, we define

$$
\mathscr{E}_{\mathrm{e}}(u)=\int_{\Omega}\left[\varepsilon|D u(x)|^{2}+W(u(x))\right] d x
$$

if $u \in \mathscr{W}^{1,2}(\Omega) ; \mathscr{E}_{e}(u)=+\infty$ otherwise. Further, it is convenient to introduce the constant

$$
c_{0}=\int_{\alpha}^{\beta} W^{\frac{1}{2}}(s) d s .
$$

Our main goal is to prove the following theorem.

Theorem I. Fix $m \in \mathbb{R}$ such that $\alpha|\Omega| \leqq m \leqq \beta|\Omega|$, and suppose that the function $u_{\varepsilon}$ is, for every $\varepsilon>0$, a solution of the variational problem

$$
\mathscr{E}_{e}\left(u_{\varepsilon}\right)=\min \left\{\mathscr{E}_{\varepsilon}(u): u \in L^{1}(\Omega), \quad u \geqq 0, \int_{\Omega} u d x=m\right\} .
$$

If $\left(\varepsilon_{h}\right)$ is a sequence of positive numbers such that $\varepsilon_{h}$ converges to zero and $\left(u_{\varepsilon_{h}}\right)$ converges to a function $u_{0}$ in $L^{1}(\Omega)$ as $h \rightarrow+\infty$, then

(i) $W\left(u_{0}(x)\right)=0$ (i.e. $u_{0}(x)=\alpha$ or $u_{0}(x)=\beta$ ) for almost all $x \in \Omega$;

(ii) the set $E=\left\{x \in \Omega: u_{0}(x)=\alpha\right\}$ is a solution of the variational problem

$$
P_{\Omega}(E)=\min \left\{P_{\Omega}(F): F \leqq \Omega,|F|=\frac{\beta|\Omega|-m}{\beta-\alpha}\right\} ;
$$

(iii) $\lim _{h \rightarrow+\infty} \varepsilon_{h}^{-\frac{1}{h}} \mathscr{E}_{\varepsilon_{h}}\left(u_{\varepsilon_{h}}\right)=2 c_{0} P_{\Omega}(E)$

We postpone to the end of this section some remarks about the statement of Theorem I.

The proof of Theorem I relies on the following two propositions; these propositions, as we shall see in section 3, are of interest in themselves.

Proposition 1. Let $\left(v_{\varepsilon}\right)_{\varepsilon \geq 0}$ be a family of functions in $L^{1}(\Omega)$ such that $v_{\varepsilon}$ converges to $v_{0}$ in $L^{1}(\Omega)$ as $\varepsilon \rightarrow 0^{+}$. 
(i) If

$$
\liminf _{\varepsilon \rightarrow 0^{+}} \varepsilon^{-\frac{1}{2}} \mathscr{E}_{\varepsilon}\left(v_{\varepsilon}\right)<+\infty,
$$

then $W\left(v_{0}(x)\right)=0$ for almost all $x \in \Omega$.

(ii) If $W\left(v_{0}(x)\right)=0$ for almost all $x \in \Omega$, then

$$
P_{\Omega}(E) \leqq \frac{1}{2 c_{0}} \liminf _{\varepsilon \rightarrow 0^{+}} \varepsilon^{-\frac{1}{2}} \mathscr{E}_{\varepsilon}\left(v_{\varepsilon}\right),
$$

where $E=\left\{x \in \Omega: v_{0}(x)=\alpha\right\}$.

Proposition 2. Let $A$ be an open subset of $\mathbb{R}^{n}$ with $\partial A$ a non-empty, compact, smooth hypersurface and $\mathscr{H}_{n-1}(\partial A \cap \partial \Omega)=0$. Define the function $v_{0}: \Omega \rightarrow \mathbb{R}$ by

$$
v_{0}(x)=\alpha \quad \text { if } x \in A \cap \Omega, \quad v_{0}(x)=\beta \quad \text { if } x \in\left(\mathbb{R}^{n} \backslash A\right) \cap \Omega .
$$

Then there is a family $\left(v_{\varepsilon}\right)_{\varepsilon>0}$ of Lipschitz continuous function on $\Omega$ such that $v$ converges to $v_{0}$ in $L^{1}(\Omega)$ as $\varepsilon \rightarrow 0^{+}, \alpha \leqq v_{\varepsilon} \leqq \beta$ for every $\varepsilon>0$, and

(i) $\int_{\Omega} v_{\varepsilon} d x=\int_{\Omega} v_{0} d x=\alpha|A \cap \Omega|+\beta|\Omega \backslash A| \quad \forall \varepsilon>0$,

(ii) $\frac{1}{2 c_{0}} \limsup _{\varepsilon \rightarrow 0^{+}} \varepsilon^{-\frac{1}{8} \mathscr{E}_{\delta}}\left(v_{\varepsilon}\right) \leqq P_{\Omega}(A)$.

Proof of Proposition 1. (i) Let us choose a sequence $\left(\varepsilon_{h}\right)$ of positive numbers, converging to zero as $h \rightarrow+\infty$, such that $\left(v_{\varepsilon_{h}}\right)$ converges pointwise to $v_{0}$ almost everywhere in $\Omega$ and

$$
\lim _{h \rightarrow+\infty} \mathscr{E}_{\varepsilon_{h}}\left(v_{\varepsilon_{h}}\right)=0
$$

Then, by Fatou's Lemma,

$$
\begin{aligned}
\int_{\Omega} W\left(v_{0}(x) d x\right. & \leqq \liminf _{h \rightarrow+\infty} \int_{\Omega} W\left(v_{\varepsilon_{h}}(x)\right) d x \\
& \leqq \liminf _{h \rightarrow+\infty} \mathscr{E}_{\varepsilon_{h}}\left(v_{\varepsilon_{h}}\right)=0 .
\end{aligned}
$$

Since $W \geqq 0$, (i) is proved.

(ii) It is not restrictive to assume $\mathscr{E}_{\varepsilon}\left(v_{\varepsilon}\right)<+\infty$ (hence $v_{\varepsilon} \in \mathscr{W}^{1,2}(\Omega)$ ) and $\alpha \leqq v_{\varepsilon} \leqq \beta$ for every $\varepsilon>0$. In fact, by considering the truncated functions

$$
\tilde{v}_{\varepsilon}=\max \left\{\alpha, \min \left\{v_{\varepsilon}, \beta\right\}\right\},
$$

we conclude that $\tilde{v}_{\varepsilon}$ converges in $L^{1}(\Omega)$ to $v_{0}$ as $\varepsilon \rightarrow 0^{+}$, and $\mathscr{E}_{\varepsilon}\left(\tilde{v}_{\varepsilon}\right) \leqq \mathscr{E}_{\varepsilon}\left(v_{\varepsilon}\right)$ for every $\varepsilon>0$.

Define

$$
\phi(t)=\int_{0}^{t} W^{\frac{1}{2}}(s) d s, \quad w_{\varepsilon}(x)=\phi\left(v_{\varepsilon}(x)\right)
$$


for $t \geqq 0, \varepsilon \geqq 0, x \in \Omega$. Since the $v_{\varepsilon}$ are equibounded and $\phi$ is of class $C^{1}$, $w_{\varepsilon}$ converges to $w_{0}$ in $L^{1}(\Omega)$ as $\varepsilon \rightarrow 0^{+}$, so the lower semicontinuity (1) yields

$$
\int_{\Omega}\left|D w_{0}\right| \leqq \liminf _{\varepsilon \rightarrow 0^{+}} \int_{\Omega}\left|D w_{\varepsilon}\right|
$$

Next, by the Fleming-Rishel formula (4) and the hypothesis $v_{0}(x)=\alpha$ or $v_{0}(x)=\beta$ for almost all $x \in \Omega$,

$$
\begin{aligned}
\int_{\Omega}\left|D w_{0}\right| & =\int_{\mathbf{R}} P_{\Omega}\left(\left\{x \in \Omega: \phi\left(v_{0}(x)\right)>t\right\}\right) d t \\
& =(\phi(\beta)-\phi(\alpha)) P_{\Omega}(E)=c_{0} P_{\Omega}(E) .
\end{aligned}
$$

On the other hand, $v_{\varepsilon} \in \mathscr{W}^{1,2}(\Omega)$ implies that $D w_{\varepsilon}(x)=\phi^{\prime}\left(v_{\varepsilon}(x)\right) D v_{\varepsilon}(x)$ (cf. Marcus \& Mizel [18]); hence

$$
\begin{aligned}
\int_{\Omega}\left|D w_{\varepsilon}\right| d x & =\int_{\Omega}\left|W^{\frac{1}{2}}\left(v_{\varepsilon}(x)\right)\right|\left|D v_{\varepsilon}(x)\right| d x \\
& \leqq \frac{1}{2} \int_{\Omega}\left[\varepsilon^{\frac{1}{2}}\left|D v_{\varepsilon}\right|^{2}+\varepsilon^{-\frac{1}{2}} W\left(v_{\varepsilon}\right)\right] d x \\
& =\frac{1}{2} \varepsilon^{-\frac{1}{6}} \mathscr{E}_{\varepsilon}\left(v_{\varepsilon}\right) .
\end{aligned}
$$

By inserting (25) and (26) in (24), (ii) is proved.

Proof of Proposition 2. Let us define the function $h$ as in Lemma 4 and the function $\chi_{0}: \mathbb{R} \rightarrow \mathbb{R}$ by

$$
\chi_{0}(t)=\alpha \quad \text { if } t<0, \quad \chi_{0}(t)=\beta \quad \text { if } t \geqq 0,
$$

so that $v_{0}(x)=\chi_{0}(h(x))$. We shall construct $\left(v_{\varepsilon}\right)$ using $\left(\chi_{\varepsilon}\right)$ to approximate $\chi_{0}$, with $\chi_{\varepsilon}$ obtained using a construction involving the ordinary differential equation $\varepsilon \chi_{\varepsilon}^{\prime 2}=\sqrt{\varepsilon}+W\left(\varkappa_{\varepsilon}\right)$.

For clarity, let us explain briefly why we choose this equation. Our aim is to approximate the two-valued function $\chi_{0}$ by a Lipschitz continuous function $\chi_{\varepsilon}$ which interpolates between $\alpha$ and $\beta$ and, at the same time, minimizes

$$
\int\left[\varepsilon \chi_{\varepsilon}^{2}+W\left(\chi_{\varepsilon}\right)\right] d t,
$$

the one-dimensional analogue of $\mathscr{E}_{\varepsilon}$. The corresponding Euler equation is $2 \varepsilon \chi_{\varepsilon}^{\prime \prime}=W^{\prime}\left(\chi_{\varepsilon}\right)$; multiplying by $\chi_{\varepsilon}^{\prime}$ and integrating, we obtain $\varepsilon \chi^{\prime 2}=c_{\varepsilon}+W\left(\chi_{\varepsilon}\right)$. The constant $c_{\varepsilon}$ cannot be set equal to zero: indeed, in this case, if $\chi_{\varepsilon}\left(t_{0}\right)=\alpha$ or $\chi_{\varepsilon}\left(t_{0}\right)=\beta$ for some $t_{0} \in \mathbb{R}$, then $\chi_{\varepsilon}$ would be constant. On the other hand, we need $c_{\varepsilon} \gg \varepsilon$ to make $\chi_{\varepsilon}$ fill the gap between $\alpha$ and $\beta$ as quickly as possible (note that $\chi_{\varepsilon}^{\prime 2} \geqq c_{\varepsilon} / \varepsilon$ ), and for that reason we choose $c_{\varepsilon}=\sqrt{\varepsilon}$.

To begin the explicit construction of $\chi_{\varepsilon}$, fix $\varepsilon>0$ and define, for $\alpha \leqq t \leqq \beta$,

$$
\psi_{\varepsilon}(t)=\int_{a}^{t}\left(\frac{\varepsilon}{\sqrt{\varepsilon}+W(s)}\right)^{\frac{1}{2}} d s
$$


and

$$
\eta_{\varepsilon}=\psi_{\varepsilon}(\beta)
$$

In addition, let $\phi_{\varepsilon}:\left[0, \eta_{\varepsilon}\right] \rightarrow[\alpha, \beta]$ denote the inverse of $\psi_{\varepsilon}$. Since $W$ is nonnegative,

$$
0<\eta_{\varepsilon} \leqq \varepsilon^{\frac{1}{4}}(\beta-\alpha)
$$

and, since $W$ is continuous, $\phi_{\varepsilon}$ is of class $C^{1}$ and

$$
\varepsilon^{\frac{1}{2}} \phi_{\varepsilon}^{\prime}(t)=\left(\sqrt{\varepsilon}+W\left(\phi_{\varepsilon}(t)\right)\right)^{\frac{1}{2}}
$$

for $0 \leqq t \leqq \eta_{\varepsilon}$.

We now extend the definition of $\phi_{\varepsilon}$ to the entire real line by setting

$$
\phi_{\varepsilon}(t)=\alpha \quad \text { for } t<0, \quad \phi_{\varepsilon}(t)=\beta \quad \text { for } t>\eta_{\varepsilon},
$$

so that $\phi_{\varepsilon}$ becomes a Lipschitz continuous function on $\mathbb{R}$. Note that, for every $t \in \mathbb{R}, \quad \phi_{\varepsilon}(t) \leqq \chi_{0}(t)$ and $\phi_{\varepsilon}\left(t+\eta_{\varepsilon}\right) \geqq \chi_{0}(t)$. Thus, there is a $\delta_{\varepsilon} \in\left[0, \eta_{\varepsilon}\right]$ such that

$$
\int_{\Omega} \phi_{\varepsilon}\left(h(x)+\delta_{\varepsilon}\right) d x=\int_{\Omega} \chi_{0}(h(x)) d x=\int_{\Omega} v_{0}(x) d x .
$$

Finally, we define $\chi_{\varepsilon}(t)=\phi_{\varepsilon}\left(t+\delta_{\varepsilon}\right)$ for $t \in \mathbb{R}$ and $v_{\varepsilon}(x)=\chi_{\varepsilon}(h(x))$ for $x \in \Omega$.

Each $v_{\varepsilon}$ is a Lipschitz continuous function and $\alpha \leqq v_{\varepsilon} \leqq \beta$; by Lemma 4

$$
\int_{\Omega}\left|v_{\varepsilon}-v_{0}\right| d x=\int_{\Omega}\left|\chi_{\varepsilon}(h(x))-\chi_{0}(h(x))\right||D h(x)| d x,
$$

so that the coarea formula ( $c f$. FedERER [10])

$$
\int_{\Omega} f(u(x))|D u(x)| d x=\int_{\mathrm{R}} f(t) \mathscr{H}_{n-1}(\{x \in \Omega: u(x)=t\}) d t,
$$

which holds for any Lebesgue measurable function $f$ and any Lipschitz continuous function $u$, implies that

$$
\begin{aligned}
\int_{\Omega}\left|v_{\varepsilon}-v_{0}\right| d x & =\int_{-\delta_{\varepsilon}}^{\eta_{\varepsilon}-\delta_{\varepsilon}}\left|\chi_{\varepsilon}(t)-\chi_{0}(t)\right| \mathscr{H}_{n-1}\left(S_{t} \cap \Omega\right) d t \\
& \leqq \eta_{\varepsilon}(\beta-\alpha) \sup _{|t| \leqq \eta_{\varepsilon}} \mathscr{H}_{n-1}\left(S_{t} \cap \Omega\right),
\end{aligned}
$$

where $S_{t}=\left\{x \in \mathbb{R}^{n}: h(x)=t\right\}$. Applying (27) and Lemma 4, we conclude that $v_{\varepsilon}$ converges to $v_{0}$ in $L^{1}(\Omega)$ as $\varepsilon \rightarrow 0^{+}$.

Since the thesis (i) is a direct consequence of (29), it remains to prove the estimate (ii). Let

$$
\gamma_{\varepsilon}=\sup _{|t| \leqq \eta_{\varepsilon}} \mathscr{H}_{n-1}\left(S_{t} \cap \Omega\right)
$$


We again employ the coarea formula (30), obtaining

$$
\begin{aligned}
\varepsilon^{-\frac{1}{2}} \mathscr{E}_{\varepsilon}\left(v_{\varepsilon}\right) & =\int_{\mathbb{R}}\left[\varepsilon^{\frac{1}{2}} \chi_{\varepsilon}^{\prime 2}(t)+\varepsilon^{-\frac{1}{2}} W\left(\chi_{e}(t)\right)\right] \mathscr{H}_{n-1}\left(S_{t} \cap \Omega\right) d t \\
& \leqq \gamma_{\varepsilon} \int_{-\delta_{\varepsilon}}^{\eta_{\varepsilon}-\delta_{\varepsilon}}\left[\varepsilon^{\frac{1}{2}} \phi_{\varepsilon}^{\prime 2}\left(t+\delta_{\varepsilon}\right)+\varepsilon^{-\frac{1}{2}} W\left(\phi_{\varepsilon}\left(t+\delta_{\varepsilon}\right)\right)\right] d t \\
& \leqq \gamma_{\varepsilon} \int_{0}^{\eta_{\varepsilon}}\left[\varepsilon^{\frac{1}{2}} \phi_{\varepsilon}^{\prime 2}(t)+\varepsilon^{-\frac{1}{2}}(\sqrt{\varepsilon}+W(\phi(t)))\right] d t
\end{aligned}
$$

and, recalling (28),

Since Lemma 4 implies

$$
\begin{aligned}
\varepsilon^{-\frac{1}{2}} \mathscr{E}_{\varepsilon}\left(v_{\varepsilon}\right) & \leqq \gamma_{\varepsilon} \int_{0}^{\eta_{\varepsilon}} 2\left(\sqrt{\varepsilon}+W\left(\phi_{\varepsilon}(t)\right)\right)^{\frac{1}{2}} \phi_{\varepsilon}^{\prime}(t) d t \\
& =2 \gamma_{\varepsilon} \int_{\alpha}^{\beta}(\sqrt{\varepsilon}+W(s))^{\frac{1}{2}} d s .
\end{aligned}
$$

we conclude that

$$
\lim _{\varepsilon \rightarrow 0^{+}} \gamma_{\varepsilon}=\mathscr{H}_{n-1}(\partial A \cap \Omega)=P_{\Omega}(A),
$$

$$
\underset{\varepsilon \rightarrow 0^{+}}{\limsup } \varepsilon^{-\frac{1}{2}} \mathscr{E}_{\varepsilon}\left(v_{\varepsilon}\right) \leqq 2 P_{\Omega}(A) \int_{\alpha}^{\beta} W^{\frac{1}{2}}(s) d s
$$

and Proposition 2 is proved.

Proof of Theorem I. Consider (i). Let us select, as comparison functions for $u_{\varepsilon}$, the following piecewise affine functions $v_{\varepsilon}$, depending only on the first variable $x_{1}$ :

$$
v_{\varepsilon}(x)= \begin{cases}\alpha & \text { if } x_{1} \leqq t_{0}-\sqrt{\varepsilon} \\ \frac{\beta-\alpha}{2 \sqrt{\varepsilon}}\left(x_{1}-t_{0}\right)+\frac{\beta-\alpha}{2} & \text { if } t_{0}-\sqrt{\varepsilon}<x_{1}<t_{0}+\sqrt{\varepsilon} \\ \beta & \text { if } x_{1} \geqq t_{0}+\sqrt{\varepsilon},\end{cases}
$$

with $t_{0}$ so chosen that

$$
\int_{\Omega} v_{\varepsilon} d x=m
$$

If we let $T_{\varepsilon}=\left\{x \in \Omega: t_{0}-\sqrt{\varepsilon} \leqq t_{0}+\sqrt{\varepsilon}\right\}$, we have, by the boundedness of $\Omega$, that $\left|T_{\varepsilon}\right| \leqq c_{1} \sqrt{\varepsilon}$ for every $\varepsilon>0$ and for a suitable real constant $c_{1}$, hence, by the minimality of $u_{\varepsilon}$,

$$
\begin{aligned}
& \varepsilon_{h}^{-\frac{1}{2}} \mathscr{E}_{\varepsilon_{h}}\left(u_{\varepsilon_{h}}\right) \leqq \varepsilon_{h}^{-\frac{1}{2} \mathscr{E}_{\varepsilon_{h}}}\left(v_{\varepsilon_{h}}\right)=\int_{T_{\varepsilon_{h}}}\left[\sqrt{\varepsilon_{h}}\left(\frac{\beta-\alpha}{2 \sqrt{\varepsilon_{h}}}\right)^{2}+\frac{1}{\sqrt{\varepsilon_{h}}} W\left(v_{\varepsilon_{h}}(x)\right)\right] d x \\
& \leqq c_{1}\left[\left(\frac{\beta-\alpha}{2}\right)^{2}+\max _{\alpha \leqq s \leqq \beta} W(s)\right] \text {. }
\end{aligned}
$$

Since $W$ is continuous, Proposition 1 applies and (i) is proved. 
Consider (ii). Suppose first that $0<|E|<|\Omega|$. Since

$$
|E|=\int_{\Omega}\left(\frac{\beta-u_{0}(x)}{\beta-\alpha}\right) d x=\frac{\beta|\Omega|-m}{\beta-\alpha},
$$

by Lemma 2 it suffices to verify that $P_{\Omega}(E) \leqq P_{\Omega}(A)$ for every open, bounded subset $A$ of $\mathbb{R}^{n}$, with smooth boundary, such that $\mathscr{H}_{n-1}(\partial A \cap \partial \Omega)=0$ and $|A \cap \Omega|=|E|$. Fix such a subset $A$ and note that $\partial A \cap \Omega \neq \emptyset$, because $0<|A \cap \Omega|<|\Omega|$; using Proposition 2 we construct a family $\left(v_{\varepsilon}\right)_{\varepsilon>0}$ in $\mathscr{W}^{1,2}(\Omega)$ such that

$$
\int_{\Omega} v_{\varepsilon} d x=\alpha|A \cap \Omega|+\beta|\Omega \backslash A|=\alpha|E|+\beta(|\Omega|-|E|)=m
$$

and

$$
\frac{1}{2 c_{0}} \limsup _{\varepsilon \rightarrow 0^{+}} \varepsilon^{-\frac{1}{2}} \mathscr{E}_{\varepsilon}\left(v_{\varepsilon}\right) \leqq P_{\Omega}(A) .
$$

On the other hand, by (i) and Proposition 2,

$$
P_{\Omega}(E) \leqq \frac{1}{2 c_{0}} \liminf _{h \rightarrow+\infty} \varepsilon_{h}^{-\frac{1}{2}} \mathscr{E}_{\varepsilon_{h}}\left(u_{\varepsilon_{h}}\right)
$$

and, by the minimality of $u_{\varepsilon_{h}}$,

$$
\mathscr{E}_{\varepsilon_{h}}\left(u_{\varepsilon_{h}}\right) \leqq \mathscr{E}_{\varepsilon_{h}}\left(v_{\varepsilon_{h}}\right) \quad \forall h \in \mathbb{N}
$$

It is now obvious that (32), (33) and (31) yield $P_{\Omega}(E) \leqq P_{\Omega}(A)$. It remains to consider the cases $|E|=|\Omega|$ and $|E|=0$, which are trivial. For instance, in the former case we have $m=\alpha|\Omega|$, so that

$$
0 \leqq \min \left\{P_{\Omega}(F): F \leqq \Omega,|F|=|\Omega|\right\} \leqq P_{\Omega}(\Omega)=0 ;
$$

but $|\Omega \backslash E|=0$ implies that $P_{\Omega}(E)=P_{\Omega}(\Omega)=0$.

Finally, let us prove (iii). By taking into account (32), (33) and (31), we find that

$$
2 c_{0} P_{\Omega}(E) \leqq \liminf _{h \rightarrow+\infty} \varepsilon_{h}^{-\frac{1}{2}} \mathscr{E}_{\varepsilon_{h}}\left(u_{\varepsilon_{h}}\right) \leqq \limsup _{h \rightarrow+\infty} \varepsilon_{h}^{-\frac{1}{2}} \mathscr{E}_{\varepsilon_{h}}\left(u_{\varepsilon_{h}}\right) \leqq 2 c_{0} P_{\Omega}(A)
$$

for any open, bounded subset $A$ of $\mathbb{R}^{n}$, with smooth boundary, such that $\mathscr{H}_{n-1}(\partial A \cap \partial \Omega)=0$ and $|A \cap \Omega|=|E|$, provided that $0<|E|<|\Omega|$. Applying Lemma 2 with $\lambda=\limsup _{h \rightarrow+\infty} \varepsilon_{h}^{-\frac{1}{2}} \mathscr{E}_{\varepsilon_{h}}\left(u_{\varepsilon_{h}}\right)$ in conjunction with (ii), we immediately obtain (iii). The proof of (iii) in the cases $|E|=|\Omega|$ and $|E|=0$ is straightforward. For instance, in the former case $m=\alpha|\Omega|$, hence $u_{\varepsilon}=\alpha$ and $\mathscr{E}_{\varepsilon}\left(u_{\varepsilon}\right)=0$ for every $\varepsilon>0$, while $P_{\Omega}(E)=0$. The proof of Theorem $I$ is now complete.

Let us conclude this section by a few comments and remarks about the statement of Theorem I. One may ask whether the convergence in $L^{1}(\Omega)$ of some sequences $\left(u_{\varepsilon_{h}}\right)$ can be guaranteed in advance. In the following proposition we show 
that the answer in "yes" if either one has an a priori $L^{\infty}$ bound ${ }^{4}$ on the $u_{\varepsilon}$, or $W$ has a (reasonable) polynomial behavior at infinity.

Proposition 3. Fix $m \in \mathbb{R}$ such that $\alpha|\Omega| \leqq m \leqq \beta|\Omega|$, and suppose that the function $u_{\varepsilon}$ is, for every $\varepsilon>0$, a solution of the variational problem

Suppose that

$$
\mathscr{E}_{\varepsilon}\left(u_{\varepsilon}\right)=\min \left\{\mathscr{E}_{\varepsilon}(u): u \in L^{1}(\Omega), u \geqq 0, \int_{\Omega} u d x=m\right\} .
$$

(a) there exists $M>0$ such that $u_{\varepsilon}(x) \leqq M$ for every $\varepsilon>0$ and $x \in \Omega$; or

(b) there exist $t_{0}>0, c_{1}>0, c_{2}>0, k>2$ such that

$$
c_{1} t^{k} \leqq W(t) \leqq c_{2} t^{k} \quad \forall t \geqq t_{0} .
$$

Then, there exists a sequence $\left(\varepsilon_{h}\right)$ of positive numbers such that $\varepsilon_{h}$ converges to zero and $\left(u_{\varepsilon_{h}}\right)$ converges to a function $u_{0}$ in $L^{1}(\Omega)$ as $h \rightarrow+\infty$.

Proof. Let $\phi$ be the primitive function of $W^{\frac{1}{2}}$ constructed in the proof of Proposition 2 and define $v_{\varepsilon}(x)=\phi\left(u_{\varepsilon}(x)\right)$. First, we shall prove that the family $\left(v_{\varepsilon}\right)_{\varepsilon>0}$ is bounded in $L^{1}(\Omega)$.

This is obvious when (a) holds. If (b) holds, it is not restrictive to assume that $t_{0} \geqq 1$, and we easily have that

$$
\phi(t) \leqq \int_{0}^{t_{0}} W^{\frac{1}{2}}(s) d s+\frac{2 \sqrt{c_{2}}}{k+2} t^{k / 2+1} \quad \forall t \geqq t_{0} .
$$

Moreover, $k \geqq 2$ implies that $\frac{k}{2}+1 \leqq k$; hence

$$
\phi(t) \leqq c_{3}+c_{4} W(t) \quad \forall t \geqq 0
$$

for some real constants $c_{3}$ and $c_{4}$. Then,

$$
\begin{aligned}
\int_{\Omega} v_{\varepsilon} d x & \leqq c_{3}|\Omega|+c_{4} \int_{\Omega} W\left(u_{\varepsilon}(x)\right) d x \\
& \leqq c_{3}|\Omega|+c_{4} \mathscr{E}_{\varepsilon}\left(u_{\varepsilon}\right),
\end{aligned}
$$

so, by recalling the proof of (i) in Theorem I, we conclude that $\left(v_{\varepsilon}\right)$ is bounded in $L^{1}(\Omega)$.

On the other hand, by recalling (26) and again the proof of (i) in Theorem I, we see that

$$
\int_{\Omega}\left|D v_{\varepsilon}\right| d x \leqq \frac{1}{2} \varepsilon^{-\frac{1}{2}} \mathscr{E}_{\varepsilon}\left(u_{\varepsilon}\right) \leqq c_{5} \quad \forall \varepsilon>0
$$

for a suitable real constant $c_{5}>0$, so the compactness (2) yields that there is a sequence $\left(\varepsilon_{h}\right)$ of positive numbers converging to zero such that $\left(v_{\varepsilon_{h}}\right)$ converges in $L^{1}(\Omega)$ to a function $v_{0}$.

${ }^{4} C f$. Gurtin \& Matano [17]. 
We now return to the functions $u_{\varepsilon}$. Let $\psi$ be the inverse function of $\phi$ and define $u_{0}(x)=\psi\left(v_{0}(x)\right)$. Let (a) hold. Since $\psi$ is bounded and uniformly continuous on bounded subsets of $\left[0,+\infty\left[\right.\right.$, it follows easily that $u_{\varepsilon_{h}}=\psi \circ v_{\varepsilon_{h}}$ converges in $L^{1}(\Omega)$ to $u_{0}$ as $h \rightarrow+\infty$ and Proposition 3 is proved.

If (b) holds, then $\phi^{\prime}(t) \geqq \sqrt{c_{1}} t_{0}^{k / 2}$ for every $t \geqq t_{0}$; hence $\psi$ is Lipschitz continuous on $\left[\phi\left(t_{0}\right),+\infty[\right.$ and uniformly continuous on the entire real line. It follows that $u_{\varepsilon_{h}}=\psi \circ v_{\varepsilon_{h}}$ converges in measure on $\Omega$ to $u_{0}$ as $h \rightarrow+\infty$; since

$$
\begin{aligned}
\int_{\Omega} u_{\varepsilon_{h}}^{k} d x & \leqq \int_{\Omega} t_{0}^{k} d x+\frac{1}{c_{1}} \int_{\Omega} W\left(u_{\varepsilon_{h}}(x)\right) d x \\
& \leqq t_{0}^{k}|\Omega|+\frac{1}{c_{1}} \mathscr{E}_{\varepsilon_{h}}\left(u_{\varepsilon_{h}}\right),
\end{aligned}
$$

we conclude as above that $\left(u_{\varepsilon_{h}}\right)$ is bounded in $L^{k}(\Omega)$ with $k \geqq 2$. Hence, by a classical theorem of measure theory, $\left(u_{\varepsilon_{h}}\right)$ actually converges in $L^{1}(\Omega)$ to $u_{0}$, and this completes the proof of Proposition 3.

Some further remarks concerning Theorem I:

(a) The assumption that $\partial \Omega$ is Lipschitz continuous can be weakened but not replaced by $\mathscr{H}_{n-1}(\partial \Omega)<+\infty$ (cf. (3) and the proof of Lemma 1).

(b) The proof of Theorem I can be repeated with only minor changes when $W$ is defined on the open positive real line with $\lim _{t \rightarrow 0^{+}} W(t)=+\infty$, or when $W$ is defined on the entire real line with the condition $u \geqq 0$ removed.

(c) The same is true for the constrained variational problem

$$
\min \left\{\mathscr{E}_{\varepsilon}(u): u \in L^{1}(\Omega), a \leqq u \leqq b, \int_{\Omega} u=m\right\}
$$

where $a, b$ are fixed positive numbers with $a<\alpha, b<\beta$. Note that, in this case, the compactness of $\left(u_{\varepsilon}\right)$ is automatically verified because of Proposition 3 . In a forthcoming paper Gurtin \& MATaNo [17] give some conditions on $W$ ensuring that all solutions $u_{\varepsilon}$ of the original variational problem lie in a strip $a \leqq u \leqq b$.

(d) It is also possible to study, by the technique of Theorem I, the asymptotic behavior, as $\varepsilon \rightarrow 0^{+}$, of the variational problem obtained by adding a perturbation $\varepsilon^{\frac{1}{2}} T(u)$, continuous in $L^{1}(\Omega)$, to $\mathscr{E}_{\varepsilon}(u)$ ( $c f$. the next section).

(e) The proof of Theorem $I$ is entirely variational: no regularity is assumed for $W$, so that, in general, there is no Euler-Lagrange equation for $u_{\varepsilon}$. On the other hand. it is easy to verify, by direct methods, that the functionals $\mathscr{E}_{\varepsilon}$ attain their minimum value in the variational problems considered above.

\section{Relation to $\boldsymbol{\Gamma}$-convergence theory}

In this section we will show how the content of the previous sections might be placed within the general framework of variational convergence. This is only a first attempt: the subject deserves a more careful and deeper treatment (see the final remark). 
Let $\Omega$ be an open, bounded subset of $\mathbb{R}^{n}$ with Lipschitz continuous boundary. A family $\left(F_{\varepsilon}\right)_{e>0}$ of real-extended functionals defined on $L^{1}(\Omega)$ is said to $\Gamma$-converge as $\varepsilon \rightarrow 0^{+}$to a functional $F_{0}$ in a fixed point $u \in L^{1}(\Omega)$ if the following two conditions are fulfilled:

(a) $\forall\left(u_{\varepsilon}\right)_{\varepsilon>0}: \lim _{\varepsilon \rightarrow 0^{+}} \int_{\Omega}\left|u_{\varepsilon}-u\right| d x=0 \Rightarrow F_{0}(u) \leqq \liminf _{\varepsilon \rightarrow 0^{+}} F_{\varepsilon}\left(u_{\varepsilon}\right)$;

(b) $\exists\left(u_{\varepsilon}\right)_{\varepsilon>0}: \lim _{\varepsilon \rightarrow 0^{+}} \int_{\Omega}\left|u_{\varepsilon}-u\right| d x=0 \quad$ and $\quad F_{0}(u) \geqq \limsup _{\varepsilon \rightarrow 0^{+}} F_{\varepsilon}\left(u_{\varepsilon}\right)$.

There is a large literature on $\Gamma$-convergence, beginning with the pioneering works of DE GIORGI [8], [9]. We refer the interested reader to the comprehensive account of AтTOUCH [4], noting that in his terminology $\Gamma$-convergence is referred to as epi-convergence.

The following abstract proposition, whose proof is quite simple, explains why $\Gamma$-convergence is convenient in the asymptotic analysis of variational problems.

Proposition 4. Suppose that $F_{\varepsilon} \Gamma$-converges as $\varepsilon \rightarrow 0^{+}$to $F_{0}$ at every point of $L^{1}(\Omega)$. For every $\varepsilon>0$, let $u_{\varepsilon}$ be a minimum point of $F_{\varepsilon}$ in $L^{1}(\Omega)$. If $u_{\varepsilon}$ converges in $L^{1}(\Omega)$ to a function $u_{0}$ as $\varepsilon \rightarrow 0^{+}$, then $u_{0}$ is a minimum point of $F_{0}$ in $L^{1}(\Omega)$ and $\lim _{\varepsilon \rightarrow 0^{+}} F_{\varepsilon}\left(u_{\varepsilon}\right)=F_{0}\left(u_{0}\right)$.

We now return to the results of Section 2. Define

$$
F_{\varepsilon}(u)=\varepsilon^{-\frac{1}{2}} \mathscr{E}_{\varepsilon}(u)=\int_{\Omega}\left[\varepsilon^{\frac{1}{2}}|D u|^{2}+\varepsilon^{-\frac{1}{2}} W(u)\right] d x
$$

$\left(F_{s}(u)=+\infty\right.$ if $\left.u \notin \mathscr{W}^{\frac{1}{2}}(\Omega)\right)$, and

$$
I_{m}(u)=0 \quad \text { if } \int_{\Omega} u d x=m, \quad I_{m}(u)=+\infty \quad \text { if } \int_{\Omega} u d x \neq m,
$$

and finally

$$
F_{0}(u)=\left\{\begin{array}{rr}
2 c_{0} \int_{\Omega}|D u| & \begin{array}{r}
\text { if } u \in B V(\Omega) \text { and } W(u(x))=0 \\
\text { for almost every } x \in \Omega
\end{array} \\
+\infty & \text { otherwise }
\end{array}\right.
$$

Then it is easy to verify that Propositions 1 and 2 are equivalent to asserting that, as $\varepsilon \rightarrow 0^{+}, F_{\varepsilon}+I_{m} \quad \Gamma$-converges to $F_{0}+I_{m}$ at every point $u \in L^{1}(\Omega)$ except for those which satisfy

(i) $\int_{\Omega} u d x=m$;

(ii) $W(u(x))=0$ for almost every $x \in \Omega$;

(iii) there is no open subset $A$ of $\mathbb{R}^{n}$ with non-empty, compact, smooth boundary such that $\mathscr{H}_{n-1}(\partial A \cap \partial \Omega)=0$ and $\{x \in \Omega: u(x)=\alpha\}=A \cap \Omega$.

Actually, it can be proved rather easily, using Lemma 2, that $\Gamma$-convergence really occurs at all points of $L^{1}(\Omega)$, so that Theorem I is a corollary of Proposition 4. 
Furthermore, the true advantage of the formulation in terms of $T$-convergence is that this convergence is invariant under continuous perturbations (it is enough to insure the definition of $\Gamma$-convergence given above). For example, one directly obtains, without additional computation, a theorem--analogous to Theorem I-for the asymptotic behavior, as $\varepsilon \rightarrow 0^{+}$, of the variational problem

$$
\min \left\{\mathscr{E}_{e}(u)+\varepsilon^{\frac{1}{2}} \int_{\Omega} w(x) u(x) d x: u \geqq 0, \int_{\Omega} u d x=m\right\}
$$

with $w \in L^{\infty}(\Omega)$.

The Modica-Mortola example of $\Gamma$-convergence cited in the introduction is exactly the $\Gamma$-convergence of $F_{\varepsilon}$ to $F_{0}$. Of course, this does not imply Theorem I since $I_{m}$ does not correspond to a continuous perturbation.

We conclude with a remark about the asymptotic $\Gamma$-expansion:

$$
\begin{aligned}
E_{\varepsilon}(u) & =\int_{\Omega}\left[\varepsilon|D u|^{2}+W_{0}(u)\right] d x \\
& =\int_{\Omega} W_{0}^{* *}(u) d x+2 c_{0} \varepsilon^{\frac{1}{2}} \int_{\Omega}|D u|+\ldots,
\end{aligned}
$$

which holds in the sense

and

$$
\min _{u \in V} \int_{\Omega} W_{0}^{* *}(u(x)) d x=\min _{u \in V} \Gamma-\lim _{\varepsilon \rightarrow 0^{+}} E_{\varepsilon}(u)
$$

$$
\min _{u \in V} 2 c_{0} \int_{\Omega}|D u|=\min _{u \in V} \Gamma-\lim _{\boldsymbol{\Omega} \rightarrow 0^{+}} \frac{E_{\varepsilon}(u)-\int_{\Omega} W_{0}^{* *}(u) d x}{\varepsilon^{\frac{1}{2}}}
$$

where $V$ denotes the set $\left\{u \in L^{1}(\Omega): \int_{\Omega} u d x=m, \int_{\Omega} W(u(x)) d x=0\right\}$ and $W_{0}^{* *}$ is the greatest convex function less than $W_{0}$.

It should be interesting to analyze more deeply this concept of asymptotic expansion. A possible question may be to find an explicit form of the next term in the development, which is probably $\varepsilon$ multiplied by

$$
\begin{aligned}
\Gamma-\lim _{\varepsilon \rightarrow 0^{+}} \frac{E_{\varepsilon}(u)-\int_{\Omega} W_{0}^{* *}(u) d x-2 c_{0} \varepsilon^{\frac{1}{2}} \int_{\Omega}|D u|}{\varepsilon} \stackrel{?}{=} \Gamma-\lim _{\varepsilon \rightarrow 0^{+}} \int_{\Omega}\left[|D u|-\varepsilon^{-\frac{1}{2}} W^{\frac{1}{2}}(u)\right]^{2} d x,
\end{aligned}
$$

where, for the last (tentative!) equality, we might use the facts: $W_{0}(u)-W_{0}^{* *}(u)=$ $W(u)$ for $\alpha \leqq u \leqq \beta ; c_{0} \int_{\Omega}|D u|=\int_{\Omega}|D(\phi \circ u)|$ for $u \in V ; \phi^{\prime}=W^{\frac{1}{2}}$;

$$
|D(\phi \circ u)(x)|=W^{\frac{1}{2}}(u(x))|D u(x)|
$$

for any function $u \in \mathscr{W}^{1,2}(\Omega)$.

Acknowledgement. This work was carried out during visits to the Institute for Mathematics and Its Applications, University of Minnesota, Minneapolis and the Institut für Angewandte Mathematik der Universität Bonn as a guest of Sonderforschungsbereich 72. I thank the two institutions for partial support. 


\section{References}

1. N. D. Alikakos \& K. C. Shaing. On the singular limit for a class of problems modelling phase transitions. To appear.

2. F. Almgren \& M. E. Gurtin. To appear.

3. G. Anzellotti \& M. Giaquinta. Funzioni BV e tracce. Rend. Sem. Mat. Univ. Padova, 60 (1978), 1-22.

4. H. Atrouch. Variational Convergence for Functions and Operators. Appl. Math. Series, Pitman Adv. Publ. Program, Boston, London, Melbourne, 1984.

5. J. Carr, M. E. Gurtin \& M. Slemrod. Structured phase transitions on a finite interval. Arch. Rational Mech. Anal., 86 (1984), 317-351.

6. E. DE Giorgi. Su una teoria generale della misura $(r-1)$-dimensionale in uno spazio a $r$ dimensioni. Ann. Mat. Pura Appl., (4) 36 (1954), 191-213.

7. E. DE GIORGI. Nuovi teoremi relativi alle misure $(r-1)$-dimensionali in uno spazio a $r$ dimensioni. Ricerche Mat., 4 (1955), 95-113.

8. E. DE GIORGI. Sulla convergenza di alcune successioni di integrali del tipo dell'area. Rendiconti di Matematica. (4) 8 (1975), 277-294.

9. E. De Giorgi \& T. Franzoni. Su un tipo di convergenza variazionale. Atti Accad. Naz. Lincei, Rend. Cl. Sc. Mat. Fis, Natur., (8) 58 (1975), 842-850.

10. H. Federer. Geometric Measure Theory. Springer-Verlag, Berlin, Heidelberg, New York, 1968.

11. W. H. Fleming \& R. W. Rishel. An integral formula for total gradient variation. Arch. Math., 11 (1960), 218-222.

12. D. Gilbarg \& N. S. Trudinger. Elliptic Partial Differential Equations of SecondOrder. Springer-Verlag, Berlin, Heidelberg, New York, 1977.

13. E. Giustı, Minimal Surfaces and Functions of Bounded Variation. Birkhäuser Verlag, Basel, Boston, Stuttgart, 1984.

14. E. Gonzalez, U. Massari \& I. Tamanini. On the regularity of boundaries of sets minimizing perimeter with a volume constraint. Indiana Univ. Math. J., 32 (1983), 25-37.

15. M. E. Gurtin. Some results and conjectures in the gradient theory of phase transitions. Institute for Mathematics and Its Applications, University of Minnesota, preprint n. 156 (1985).

16. M. E. Gurtin. On phase transitions with bulk, interfacial, and boundary energy. Arch. Rational Mech. Anal., 96 (1986), 243-264.

17. M. E. GuRTin \& H. Matano. On the structure of equilibrium phase transitions within the gradient theory of fluids. To appear.

18. M. Marcus \& V. J. Mizel. Nemitsky Operators on Sobolev Spaces. Arch. Rational Mech. Anal., 51 (1973), 347-370.

19. U. MASSARI \& M. MirandA. Minimal Surfaces of codimension one. North-Holland Math. Studies 91, North-Holland, Amsterdam, New York, Oxford, 1984.

20. L. Modica \& S. Mortola. Un esempio di $\Gamma^{-}$-convergenza. Boll. Un. Mat. Ital., (5) 14-B (1977), 285-299.

21. L. Modica \& S. Mortola. The $\Gamma$-convergence of some functionals. Istituto Matematico "Leonida Tonelli", Università di Pisa, preprint n. 77-7 (1977).

22. A. Novick-Cohen \& L. A. SEgel. Nonlinear aspects of the Cahn-Hilliard equation. Physica, 10-D (1984), 278-298.

Dipartimento di Matematica Università di Pisa 